\title{
$\alpha 2 \delta 1$ may be a potential marker for cancer stem cell in laryngeal squamous cell carcinoma
}

\author{
Chaoping Huang ${ }^{\mathrm{a}, \mathrm{b}, 1}$, Yan $\mathrm{Li}^{\mathrm{a}, 1}$, Wei Zhao ${ }^{\mathrm{c}}$, Aobo Zhang ${ }^{\mathrm{a}}$, Cheng $\mathrm{Lu}^{\mathrm{a}}$, Zhenxiao Wang ${ }^{\mathrm{a}}$ and \\ Liangfa Liu ${ }^{\mathrm{a}, *}$ \\ ${ }^{a}$ Department of Otolaryngology and Head and Neck Surgery, Beijing Friendship Hospital, Capital Medical \\ University, Beijing 100050, China \\ ${ }^{\mathrm{b}}$ Department of Otolaryngology Head and Neck Surgery, The First Affiliated Hospital of Chengdu Medical \\ College, Chengdu 610500, Sichuan, China \\ ${ }^{\mathrm{c}}$ Key Laboratory of Carcinogenesis and Translational Research (Ministry of Education), Department of Cell \\ Biology, Peking University Cancer Hospital and Institute, Beijing 100142, China
}

\begin{abstract}
Cancer stem cells (CSCs) have the ability to dictate tumor initiation, recurrence, and metastasis. Here, we examined the expression of $\mathrm{a} \alpha 2 \delta 1^{+}$in laryngeal cancer tissues and further determined the effect of $\alpha 2 \delta 1$ on the migratory ability and tumorigenicity of laryngeal cancer cells. Immunofluorescence staining revealed that $\alpha 2 \delta 1$ was positive in $13(13 / 16,81.25 \%)$ cases in laryngeal squamous cell carcinoma (LSCC) tissues, $7(7 / 16,43.75 \%)$ cases in paracancerous tissues and only $2(2 / 16$, $12.5 \%$ ) cases in normal tumor tissues. Our quantitative RT-PCR assays further showed that $\alpha 2 \delta 1^{+}$LSCC cells expressed significantly higher levels of stem cell-associated genes and drug efflux and resistance genes versus $\alpha 2 \delta 1^{-}$cells. Sphere-forming assays demonstrated higher sphere-forming efficiency in the $\alpha 2 \delta 1^{+}$versus $\alpha 2 \delta 1^{-}$subpopulation. Our Matrigel assays showed that $\alpha 2 \delta 1^{+}$cells exhibited significantly greater invasive and migratory ability than $\alpha 2 \delta 1^{-}$cells. Furthermore, the percentage of purified $\alpha 2 \delta 1^{+}$in TU686 and TU212 cells treated cisplatin or paclitaxel was significantly higher than that of the control group. Tumor xenograft assays revealed that the tumorigenicity of $\alpha 2 \delta 1^{+}$cells was much higher than $\alpha 2 \delta 1^{-}$cells. In conclusion, a $\alpha 2 \delta 1^{+}$subpopulation with CSC-like property was present in laryngeal cancer and possessed high self-renewal activity and was sufficient for tumor growth, differentiation, migration, invasion, and chemotherapeutic resistance. They could represent a promising therapeutic target for LSCC.
\end{abstract}

Keywords: Laryngeal squamous cell carcinoma, cancer stem cells, $\alpha 2 \delta 1$

\section{Background}

Laryngeal cancer is the second most common head and neck malignancy. Globally, approximately 110,000 to 130,000 new cases are diagnosed each year [1]. In China, the crude incidence rate of laryngeal cancer was

\footnotetext{
${ }^{1}$ These authors contributed equally to this work and should be considered co-first authors.

*Corresponding author: Liangfa Liu, Department of Otolaryngology and Head and Neck Surgery, Beijing Friendship Hospital, Capital Medical University, Beijing 100050, China. Tel.: +86 013126606616; E-mail: liuliangfa301@263.net.
}

1.86/100,000 between 2008 and 2012 and the crude mortality was $1.01 / 100,000$ [2]. Mainstay treatments for laryngeal cancer include radiotherapy, chemotherapy and surgical resection; however, management of advanced laryngeal cancer is complex and a consensus therapeutic strategy has not yet emerged.

Cancer stem cells (CSCs) are implicated in tumor initiation and differentiation, and are of high selfrenewal properties, and can drive the tumorigenic process. The ability of CSCs to drive cancer initiation and progression also make them prime targets for treatments [4]. Evidence suggests that a minor population of CSCs in laryngeal cancer is extremely tu-

ISSN 1574-0153/19/\$35.00 (C) 2019 - IOS Press and the authors. All rights reserved

This article is published online with Open Access and distributed under the terms of the Creative Commons Attribution Non-Commercial License (CC BY-NC 4.0). 
morigenic and possesses the potential to differentiate into cells that are responsible for tumor propagation and relapse [4]. Laryngeal CSCs are characterized by the expression of aldehyde dehydrogenase isoform 1 (ALDH1) [5], CD133 [6], and CD44 [7,8]. However, laryngeal CSCs appear to differ considerably and may contribute to the heterogeneity of laryngeal cancer [9]. The calcium channel $\alpha 2 \delta 1$ subunit serves an important role in regulating calcium oscillation amplitude [10] and has been reported in hepatocellular carcinoma (HCC) [11]. This subpopulation of HCC cells possesses stem cell-like properties. In comparison to the non-purified cells, the invasiveness ability, self-renewal ability and the expression level of genes related to stem cells were increased in $\alpha 2 \delta 1^{+} \mathrm{HCC}$ cells $[11,12]$. Knocking down the $\alpha 2 \delta 1$ gene in combination with doxorubicin treatment compromises HCC oncogenesis [11]. Recently, Yu et al. have demonstrated that non-small cell lung cancer cells expressing $\alpha 2 \delta 1$ demonstrated CSC-like properties, and may contribute to chemoresistance [13].

Currently, no study is available on the role of $\alpha 2 \delta 1$ in laryngeal cancer. In the present study, we examined the expression of a $\alpha 2 \delta 1^{+}$in laryngeal cancer tissues and further determined the effect of $\alpha 2 \delta 1$ on the migratory ability and tumorigenicity of laryngeal cancer cells.

\section{Materials and methods}

\subsection{General design}

The current study included 3 major parts. In the first part that involved clinical samples, $\alpha 2 \delta 1$ expression was compared between primary lesions versus noncancerous adjacent tissues collected from 16 LSCC patients receiving larygectomy and no neoadjuvant therapy. The second part of the study was conducted using 2 representative laryngeal squamous cell carcinoma (LSCC) cell lines: TU212 (highly malignant) versus TU686 (less malignant). Briefly, $\alpha 2 \delta 1^{+}$cells were purified using flow cytometry from cultured cells. The following features were compared between $\alpha 2 \delta 1^{+}$and $\alpha 2 \delta 1^{-}$cells: 1) representative CSC markers (BMI1, SOX2, EPCAM, OCT4, CNTTB, KLF4, $N A N O, A B C G 2$ and $M D R 1)$; 2) sphere formation; 3) differentiation; 4) migration/invasion; 5) sensitivity to cisplatin/paclitaxel. These features were also examined after $\alpha 2 \delta 1^{+}$knockdown using shRNAs in $\alpha 2 \delta 1^{+}$ cells. In the final step of xenograft experiments with
NOD/SCID mice, the ability of $\alpha 2 \delta 1^{+}$versus $\alpha 2 \delta 1^{-}$ TU686 cells to form tumor and subsequent growth was compared using limited dilution.

\subsection{Tissue specimen acquisition}

Archived surgically resected laryngeal squamous cell carcinoma (LSCC) tissue specimens were obtained from 16 treatment-naive male patients and snap-frozen in liquid nitrogen. The study protocol was approved by the institute ethics committee of Beijing Friendship Hospital, Capital Medical University (no. 2017P2-187-01) and written informed consent was obtained from all the study subjects.

\subsection{Immunofluorescence staining}

Frozen tissues were sectioned with acryostat and fixed with methanol for 30 seconds. After blocking with 5\% nonfat milk in PBS, slides were incubated with $\alpha 2 \delta 1$ monoclonal antibody (dilutions 1:100; Catalog no. MA3-921, ThermoFisher Scientific) overnight at $4{ }^{\circ} \mathrm{C}$, followed by incubation with FITC-conjugated goat-anti-mouse IgG. In negative control, the antibody was omitted. Nuclei were counterstained with 4,6-diamidino-2-phenylindole dihydrochloride (DAPI; Polysciences, Warrington, PA, USA). The slides were mounted in $90 \%$ glycerol/PBS containing $2.5 \%$ 1,4-diazabicyclo $(2,2,2)$ octane and examined with a Leica SP5 confocal microscope (Leica, Wetzlar, Germany).

\subsection{Cells}

Human LSCC cell lines TU212 and TU686 were obtained from Shanghai Huiying Biological Technology (Shanghai, China), and were cultured in RPMI 1640 medium (Invitrogen, Grand Island, NY, USA) supplemented with $10 \%$ fetal bovine serum (FBS), $100 \mathrm{U} / \mathrm{mL}$ penicillin, and $100 \mathrm{mg} / \mathrm{mL}$ streptomycin (Invitrogen) at $37^{\circ} \mathrm{C}$ in a humidified atmosphere with $5 \% \mathrm{CO}_{2}$.

\subsection{Flow cytometry}

For isolation of $\alpha 2 \delta 1^{+}$cells, TU212 and TU686 cells were rinsed in phosphate buffered saline (PBS), and dissociated with $0.25 \%$ trypsin (Thermo Fisher Scientific, Waltham, USA). The cells were stained with FITC-conjugated $\alpha 2 \delta 1$ monoclonal antibody (dilutions: 1:100; Catalog No. MA3-921, ThermoFisher Scientific) for $30 \mathrm{~min}$ at $4^{\circ} \mathrm{C}$. Then, $\alpha 2 \delta 1^{+}$cells were 
Table 1

Primer sequences for quantitative PCR in the study

\begin{tabular}{lll}
\hline Gene & Sense & Anti-sense \\
\hline BMI1 & 5-AGCAGCAATGACTGTGATGC-3 & 5-CAGTCTCAGGTATCAACCAG-3 \\
SOX2 & 5-ACATGAACGGCTGGAGCAAC-3 & 5-AGGAAGAGGTAACCACAGGG-3 \\
EPCAM & 5-CTGAATTCTCAATGCAGGGTC-3 & 5-CCCATCTCCTTTATCTCAGCC-3 \\
OCT-4 & 5-TGGGCTCGAGAAGGATGTGGTC-3 & 5-AGTTTGAATGCATGGGAGAGC-3 \\
CTNNB & 5-TGATGGAGTTGGACATGGCC-3 & 5-CTCATACAGGACTTGGGAGG-3 \\
KLF4 & 5-AAGCCAAAGAGGGGAAGAC-3 & 5-CATCTGAGCGGGCGAATTTC-3 \\
NANOG & 5-TGCCTCACACGGAGACTGTC-3 & 5-TGCTATTCTTCGGCCAGTTG-3 \\
ABCG2 & 5-GGAGGCCTTGGGATACTTTGAA-3 & 5-GAGCTATAGAGGCCTGGGGATTAC-3 \\
MDR-1 & 5-GCCTGGCAGCTGGAAGACAAATAC-3 & 5-ATGGCCAAAATCACAAGGGTTAGC-3 \\
GAPDH & 5-GACCCCTTCATTGACCTCAAC-3 & 5-CTTCTCCATGGTGGTGAAGA-3 \\
\hline
\end{tabular}

sorted using a Facscan flow cytometer (Becton Dickinson, Mountain View, CA, USA). Furthermore, TU686 cells were treated with cisplatin $(6 \mu \mathrm{mol} / \mathrm{L})$ or paclitaxel $(0.2 \mu \mathrm{mol} / \mathrm{L})$ for 72 hours. The percentage of $\alpha 2 \delta 1^{+}$cells was then detected by flow cytometry. The results were calculated with the software FlowJo (Tree Star Inc., Ashland, Oregon) and FACSCanto II (BDBiosciences).

\subsection{Quantitative reverse transcription ( $q R T)-P C R$}

Total RNA was extracted from $\alpha 2 \delta 1^{+}$and $\alpha 2 \delta 1^{-}$ cells with Trizol reagent (Invitrogen). The mRNA levels of BMI-1, SOX2, EpCAM, OCT4, CTNNB, KLF4, NANOG, ABCG2 and MDR1 were determined by quantitative reverse transcription PCR (qRT-PCR) and normalized against $\beta$-actin. qRT-PCR was performed on an ABI7500 PCR machine using the SYBR Green PCR Master Mix (Toyobo Co. Ltd., Osaka, Japan). The primer sequences are shown in Table 1 [14-22]. Expression differences were calculated by the $2^{-\Delta \Delta \mathrm{Ct}}$ method [23]. $\beta$-actin was used as an endogenous reference.

\subsection{Plasmids}

Retroviral-based plasmids containing a short hairpin RNA (shRNA) against human $\alpha 2 \delta 1$ were purchased from Origene (Rockville, MD, USA). The U6 promoter-driven shRNA expression cassettes were transferred to the lentiviral shuttle vector plenti6 (Invitrogen). Lentiviral packaging, infection, and selection of blasticidin-resistant cell pools were performed as previously described [24]. The sequences of human $\alpha 2 \delta 1$ specific 29-mer shRNAs were as follows: shRNA53, 5'-ACTCAACTGGACAAGTGCCTTAGATGAAG-3' and shRNA56, 5'-AGATGCAAGAAGACCTTGTCA CACTGGCA-3'. Scrambled shRNA was used as a negative control.

\subsection{Sphere formation assays}

Sphere-formation assays were carried out as previously published [10]. Briefly, $\alpha 2 \delta 1^{+}$and $\alpha 2 \delta 1^{-}$ cells were cultured in DMEM supplemented with $1 \%$ methylcellulose (Sigma, St. Louis, MO, USA), B27 (Invitrogen), $20 \mathrm{ng} / \mathrm{mL}$ basic fibroblast growth factor (bFGF) (Peprotech, Rocky Hill, NJ, USA) and $20 \mathrm{ng} / \mathrm{mL}$ epidermal growth factor (EGF) (Peprotech) using a 96-well plate with ultralow attachment. The culture medium was replenished with $200 \mu \mathrm{L}$ supplementary media every other day. Spheroids $(\geqslant 100 \mu \mathrm{m})$ were counted using a microscope (Zeiss, Oberkochen, Germany) after 4 weeks. In addition, passage two $\alpha 2 \delta 1^{+}$cells and $\alpha 2 \delta 1^{+}$cells treated with shRNA53 and shRNA56 were used for sphere-formation assays where indicated.

\subsection{Cell differentiation assays}

$\alpha 2 \delta 1^{+}$cells were seeded in a Petri dish containing $10 \mathrm{~mL}$ RPMI 1640 and $10 \% \mathrm{FBS}$ and cultured at $37^{\circ} \mathrm{C}$ in a humidified incubator with $5 \% \mathrm{CO}_{2}$. After 1 week of culture, the percentage of $\alpha 2 \delta 1^{+}$cells was calculated by flow cytometry.

\subsection{Matrigel assays}

For detection of migration and invasion of $\alpha 2 \delta 1^{+}$ cells, $5 \times 10^{4}$ cells were added onto a porous membrane (pore size, $8 \mu \mathrm{m}$; BD Biosciences) that was coated with $2 \mathrm{mg} / \mathrm{mL}$ Matrigel. After 48-hour incubation at $37^{\circ} \mathrm{C}$, cells were washed three times with PBS, and fixed with $4 \%$ neutral formaldehyde prior to routine hematoxylin staining for $5 \mathrm{~min}$. The number of cells that invaded through the membrane (migration) or Matrigel (invasion) was counted in 10 representative fields at $\times 20$ magnification. Images were acquired and analyzed using SPOT imaging software (Nikon). 

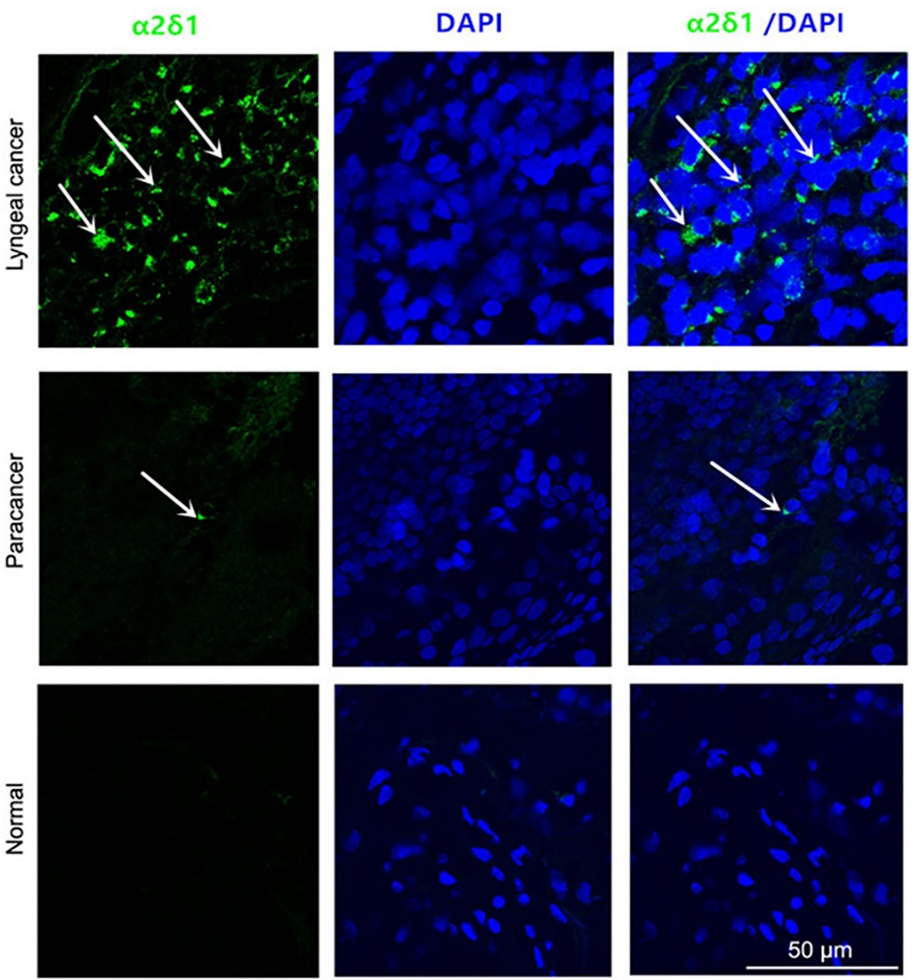

A

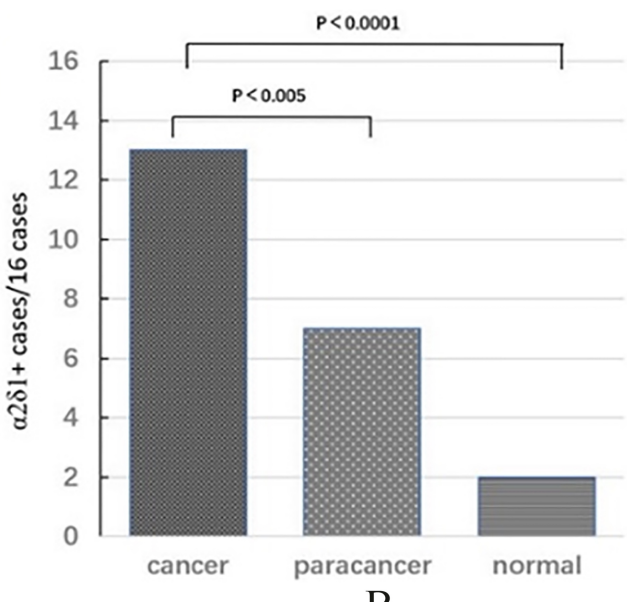

B

Fig. 1. Expression of $\alpha 2 \delta 1$ in primary LSCC tissues. (A) Representative images of immunofluorescence expression of $\alpha 2 \delta 1$ in laryngeal tissue, paracancerous tissue and normal adjacent tissue in a patient with T3N0M0 laryngeal cancer are shown. $\alpha 2 \delta 1$ (green) was stained with specific monoclonal antibody and nuclei (blue) were stained with DAPI. Arrows indicate $\alpha 2 \delta 1$-positive cells. (B) Summary of $\alpha 2 \delta 1$ expression in LSCC tissues, paracancerous tissues and normal adjacent tissues.

\subsection{Tumor xenograft assays}

Four to 6 week-old female non-obese diabetic/severe combined immunodeficient (NOD/SCID) female mice were purchased from Huafu Kang Experimental Animal Co., Ltd (Beijing, China), and maintained in a SPF facility. All animal experiments were performed in accordance with the National Institutes of Health Guide for the Care and Use of Laboratory Animals. The protocols were approved by the Animal Care and Use Committee at Peking University Cancer Hospital.

Cells were suspended in $50 \mathrm{~mL}$ in a 1:1 mixture of RPMI 1640 and Matrigel (BD Biosciences) and $10^{2}$ and $10^{3}$ cells were injected into the right and left flank of each mouse, respectively. Tumor formation was monitored weekly. Twenty weeks after inoculation, all mice were euthanized with an overdose of anesthesia(20\% urethane). Tumor volume was determined using the formula $V=L \times W^{2} \times 0.5$, where $L$ and $W$ represent the largest and the smallest diameter, respectively.

\subsection{Statistical analysis}

Data was analyzed using SPSS 17.0 software (IBM, Armonk, New York, NY, USA). Student's $t$ test, $\chi^{2}$ test and one-way analysis of variance (ANOVA) were used to analyze the differences. Tumorigenic cell frequency was calculated based on extreme limiting dilution analysis using the web tool at http://bioinf.wehi.edu.au/ software/elda/. $p<0.05$ (2-sided) was considered statistically significant.

\section{Results}

\section{1. $\alpha 2 \delta 1$ is predominantly expressed in LSCC tissues}

Immunofluorescence staining revealed abundant $\alpha 2 \delta 1^{+}$cells in LSCC tissues, but hardly any $\alpha 2 \delta 1^{+}$ cells in normal laryngeal tissues and occasional $\alpha 2 \delta 1^{+}$ cells in paracancerous tissues (Fig. 1A). Furthermore, $\alpha 2 \delta 1$ was positive in $13(13 / 16,81.25 \%)$ cases in 


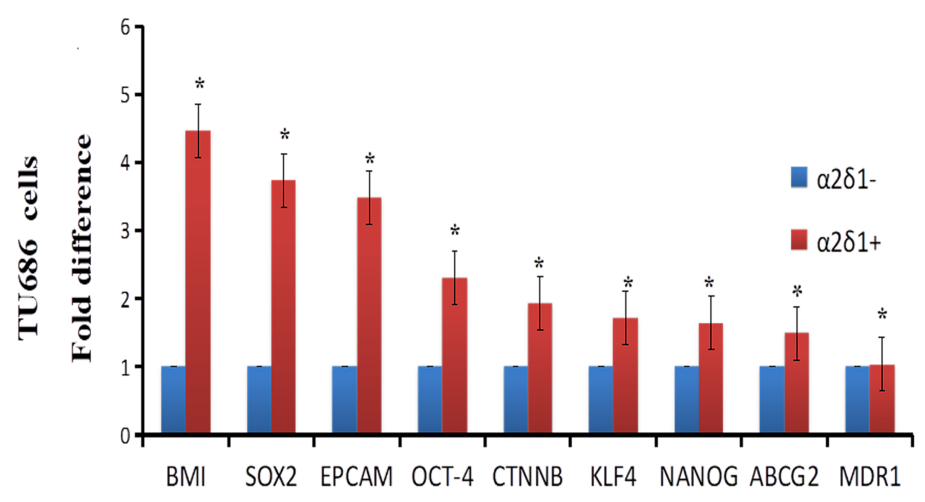

Fig. 2. Expression of stem cell and drug efflux and resistance genes in $\alpha 2 \delta 1^{-}$versus $\alpha 2 \delta 1^{+}$subpopulation of TU686 cells. qRT-PCR analysis of the expression of stem cell markers and drug-resistance-related genes in purified $\alpha 2 \delta 1^{+}$and $\alpha 2 \delta 1^{-}$subpopulations of TU686 cells. Data are presented as fold difference over $\alpha 2 \delta 1^{-}$cells for each gene. Compared with $\alpha 2 \delta 1^{-}$cells, $\alpha 2 \delta 1^{+}$cells consistently expressed 1 to 4.5 fold higher levels of BMI1, SOX2, EPCAM, OCT4, CNTTB KLF4, NANOG, ABCG2 and MDR1. *Error bars indicate SD

LSCC tissues, 7 (7/16, 43.75\%) cases in paracancerous tissues and only $2(2 / 16,12.5 \%)$ cases in normal tumor tissues (Fig. 1B).

\subsection{Stem cells-associated genes and drug efflux and resistance genes are significantly upregulated in $\alpha 2 \delta 1^{+}$TU686 cells}

We isolated $\alpha 2 \delta 1^{+}$and $\alpha 2 \delta 1^{-}$cells in TU686 cells via FACS. Our qRT-PCR assays showed that $\alpha 2 \delta 1^{+}$ cells expressed significantly higher levels of stem cell-associated genes versus $\alpha 2 \delta 1^{-}$cells: EpCAM (3.5 fold), OCT4 (2.3 fold), CNTTB1 (2.0 fold), KLF4 (1.7 fold), NANO (1.6 fold), and $A B C G 2$ (1.5 fold) (all $P<0.05)$. In addition, the $\alpha 2 \delta 1^{+}$subpopulation expressed significantly higher levels of the drug efflux transporter gene BMII (4.5 fold) and the multidrug resistance gene SOX2 (3.8 fold) compared to $\alpha 2 \delta 1^{-}$cells $(P<0.05)$ (Fig. 2)

\subsection{Self-renewal property of $\alpha 2 \delta 1^{+}$cells}

Sphere formation assays further showed that $\alpha 2 \delta 1^{+}$ cells formed a significantly higher number of spheres than $\alpha 2 \delta 1^{-}$cells $(P<0.05)$ (Fig. 3A-C). Furthermore, passage $2 \alpha 2 \delta 1^{+}$cells had significantly increased sphere-forming ability versus passage $1 \alpha 2 \delta 1^{+}$ cells (Fig. 3D and E). Moreover, knocking down $\alpha 2 \delta 1$ by $\alpha 2 \delta 1$-specific shRNAs significantly inhibited the sphere forming ability of $\alpha 2 \delta 1^{+}$cells (Fig. 3F).

\subsection{Differentiation properties of $\alpha 2 \delta 1^{+}$cells}

After purified TU212 $\alpha 2 \delta 1^{+}$cells were cultured for 1 week, the percentage of $\alpha 2 \delta 1^{+}$cells decreased from
$96.85 \%$ to $73.27 \%$; the percentage of $\alpha 2 \delta 1^{+}$TU686 cells decreased from $92.49 \%$ to $3.08 \%$ (Fig. 4), suggesting that $\alpha 2 \delta 1^{+}$cells differentiated into $\alpha 2 \delta 1^{-}$ cells.

\subsection{Migratory and invasive potential of $\alpha 2 \delta 1^{+}$cells}

Our Matrigel assays showed that $\alpha 2 \delta 1^{+}$cells exhibited significantly greater invasive ability than $\alpha 2 \delta 1^{-}$ cells (Fig. 5A-D). Furthermore, in comparison to $\alpha 2 \delta 1^{-}$cells, $\alpha 2 \delta 1^{+}$cells showed significantly greater migratory ability (Fig. 5E).

\subsection{Chemoresistance of $\alpha 2 \delta 1^{+}$cells}

We treated TU686 cells with cisplatin $(6 \mu \mathrm{mol} / \mathrm{L})$ or paclitaxel $(0.2 \mu \mathrm{mol} / \mathrm{L})$ for 72 hours. Our flow cytometric analysis showed the percentage of purified $\alpha 2 \delta 1^{+}$cells in TU686 cells treated cisplatin or paclitaxel was significantly higher than that of the control group (cisplatin: $7.87 \%$ vs. control: $1.72 \%$; paclitaxel:10.87\% vs. control: $1.72 \%$ ) (Fig. 6).

\subsection{Tumorigenesis of $\alpha 2 \delta 1^{+}$cells}

As few as $10^{2}-10^{3}$ purified $\alpha 2 \delta 1^{+}$cells from the TU212 and TU686 cell lines initiated tumor formation in almost all inoculated mice (Fig. 7A-E). By contrast, $\alpha 2 \delta 1^{-}$cells were completely non-tumorigenic or formed tiny nodules only occasionally (Fig. 7A-E). Passage $2 \alpha 2 \delta 1^{+}$and $\alpha 2 \delta 1^{-}$cells further confirmed that $\alpha 2 \delta 1^{+}$cells were consistently more tumorigenic than $\alpha 2 \delta 1^{-}$cells (Fig. 7F and G). As a matter of fact, mice inoculated with passage $2 \alpha 2 \delta 1^{-}$cells showed no 

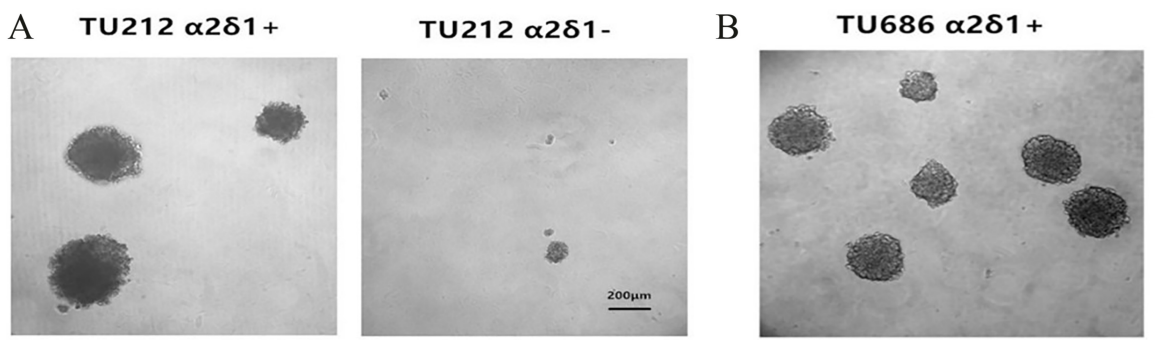

TU686 $\propto 2 \delta 1-$

$\mathrm{C}$

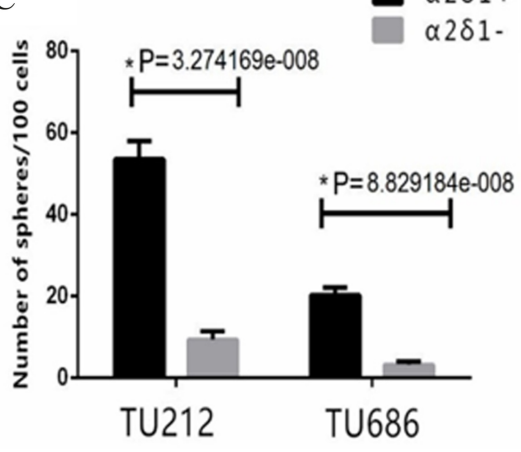

$\mathrm{E}$

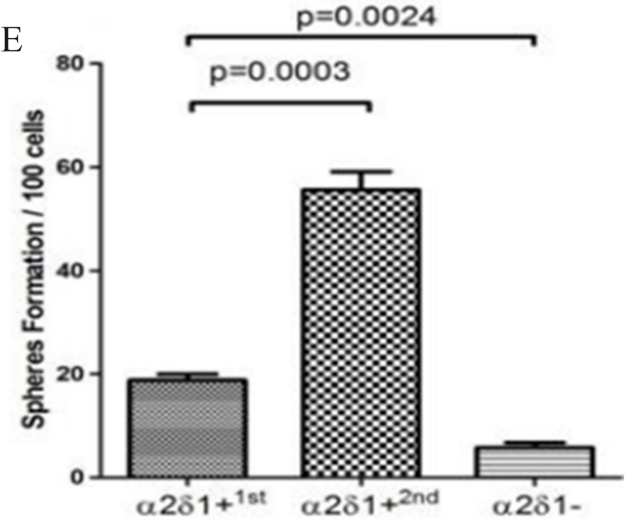

$\mathrm{D}$

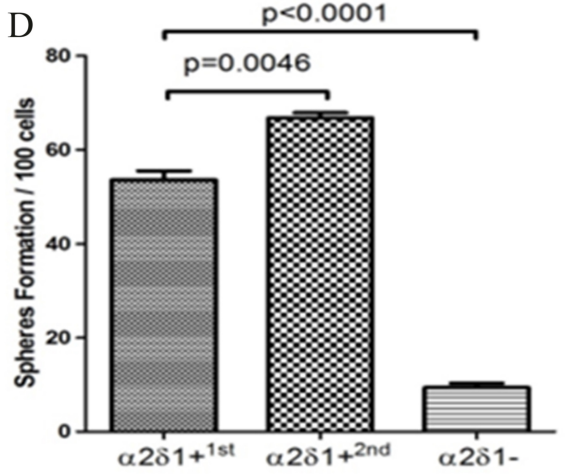

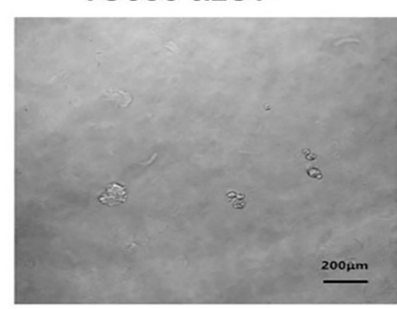

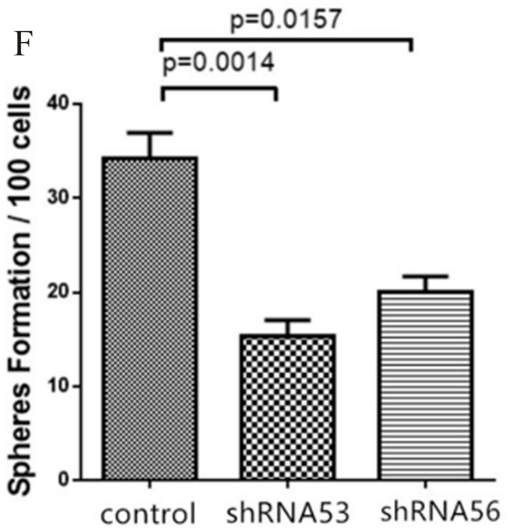

Fig. 3. Sphere-forming efficiency of $\alpha 2 \delta 1^{+}$and $\alpha 2 \delta 1^{-}$cells. (A, B) The sphere-forming ability of $\alpha 2 \delta 1^{+}$and $\alpha 2 \delta 1^{-}$cells was examined by sphere formation assays as detailed in Methods. (C) Histograms show the spheroid forming efficiency of FACS-sorted $\alpha 2 \delta 1^{+}$and $\alpha 2 \delta 1^{-}$fractions from indicated sources. The ability of the spheres formed by $\alpha 2 \delta 1^{+} \mathrm{TU} 212$ cells (D) and TU 686 cells (E) to form secondary spheroids is also shown $\left(\alpha 2 \delta 1^{+} / 2^{\text {nd }}\right.$ passage). (F) The sphere forming ability of purified $\alpha 2 \delta 1^{+}$TU212 cells was reduced when $\alpha 2 \delta 1^{+}$cells were incubated with indicated lentivirus for 4 hours, and were plated at 100 cells per well $(n=5)$. One hundred cells per well were plated $(n=5)$. Spheroids $(\geqslant 100 \mu \mathrm{m})$ were counted under a stereomicroscope. Statistical analysis was carried out using data on sphere formation in 100 cells $(\mathrm{C}, \mathrm{F})$. Data are represented as mean $\pm \mathrm{SD}$.

evident tumor formation at 12 weeks (Fig. 7F and G). Furthermore, shRNA53 and shRNA56 $\alpha 2 \delta 1^{+}$knockdown significantly suppressed the of tumor xenografts from $\alpha 2 \delta 1^{+}$TU686 cells (Fig. 7H-J).

\section{Discussion}

$\alpha 2 \delta 1$ is a voltage-gated calcium (Cav) channel subunit [25] that governs synaptic input integration, acti- vation of calcium-dependent signaling cascades, neuronal migration, neurotransmitter, and hormone release [26]. Existing evidence shows that $\alpha 2 \delta 1$ is expressed in HCC [10]. Knocking down $\alpha 2 \delta 1$ induced apoptosis of tumor-initiating cells (TIC) and inhibited tumor formation and self-renewal capacities of HCC cells. In contrast, $\alpha 2 \delta 1$ overexpression increased sphere formation. These results indicated that $\alpha 2 \delta 1^{+}$ HCC cells possess stem cell-like properties. In the current study, we found that $\alpha 2 \delta 1$ was expressed in a 


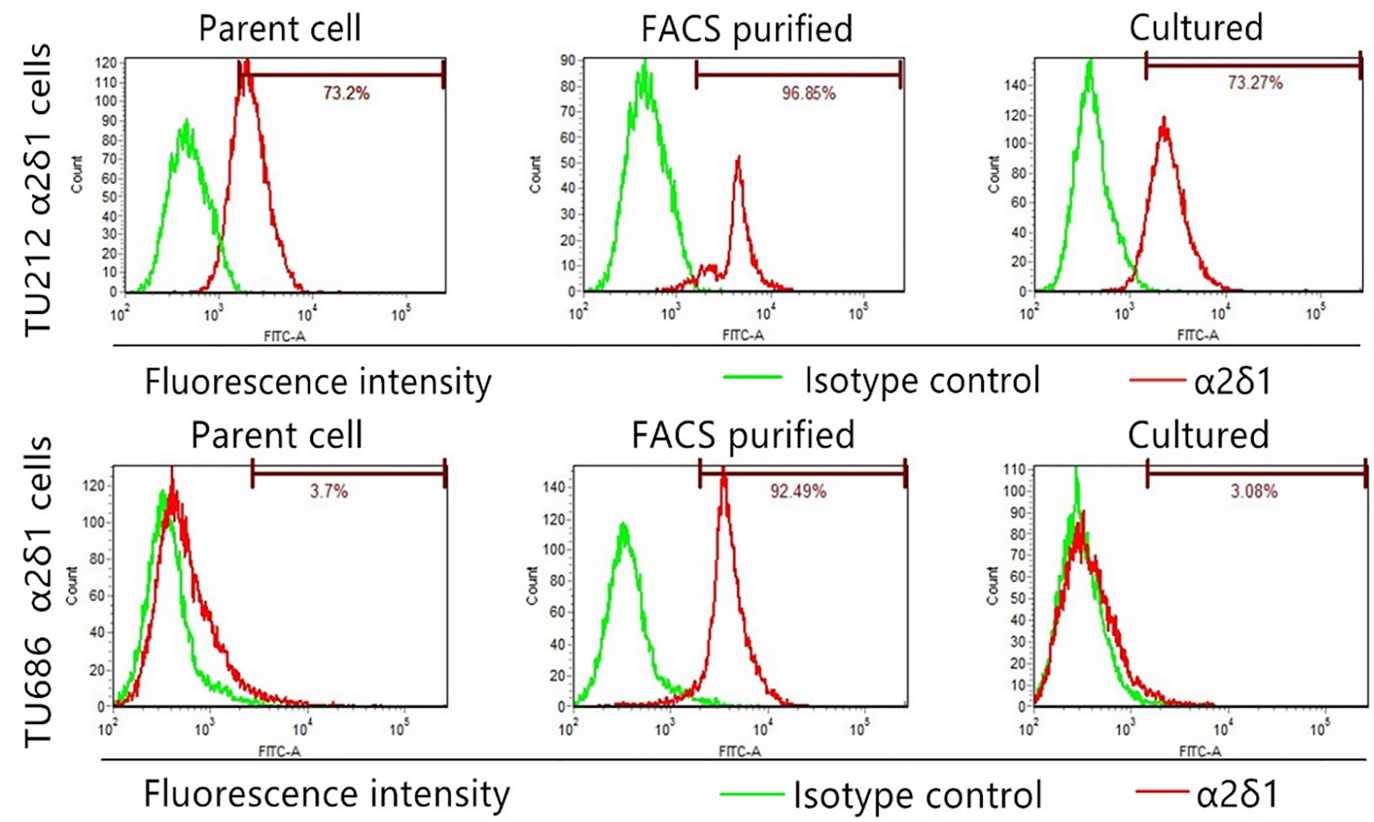

Fig. 4. Differentiation properties of $\alpha 2 \delta 1^{+}$TU212 and TU686 cells. Flow cytometry shows the percentage of $\alpha 2 \delta 1^{+}$cells in TU212 and TU686 cells, FACS-purified $\alpha 2 \delta 1^{+}$cells and purified $\alpha 2 \delta 1^{+}$cells cultured in $10 \%$ serum-containing medium for 1 week (cultured).
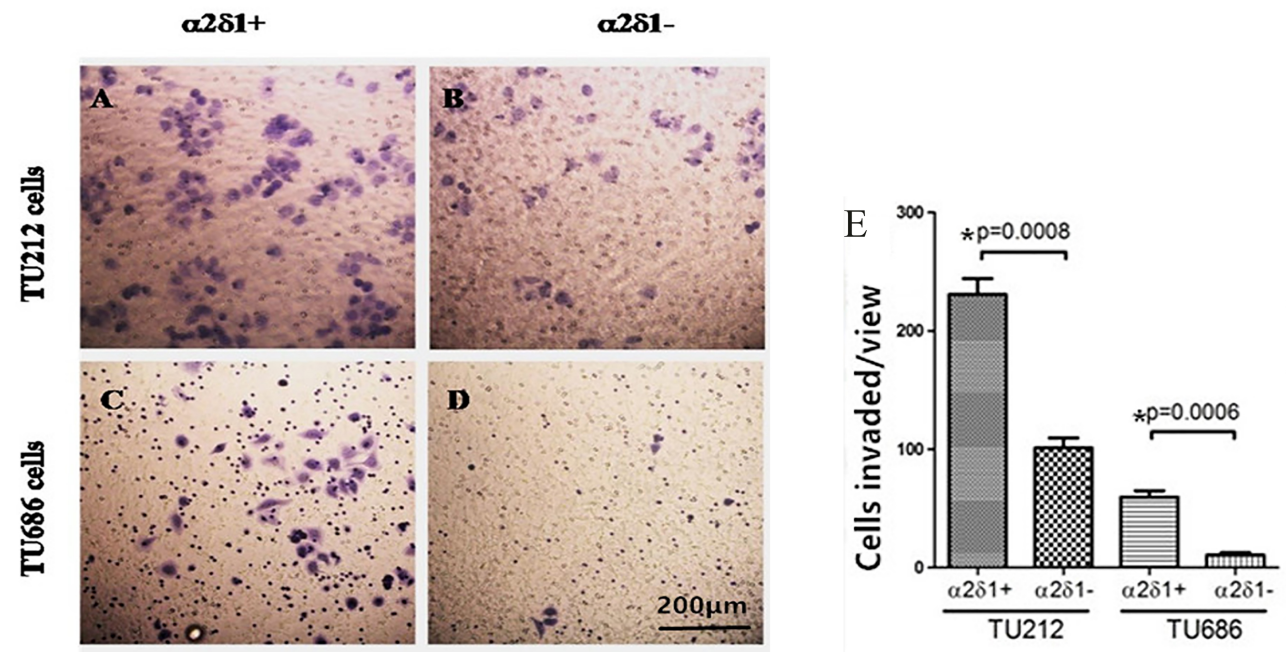

Fig. 5. Migration and invasive potential of $\alpha 2 \delta 1^{+}$cells. Sorted $\alpha 2 \delta 1^{+}$and $\alpha 2 \delta 1^{-}$cells were assayed for their invasive ability on Matrigel using a Boyden chamber assay. The invasive and migratory potential of $\alpha 2 \delta 1^{+}$cells (A, C) and $\alpha 2 \delta 1^{-}$cells (B, D) were detected. Statistical results of invasive cells were calculated (E). Data represent the mean $\pm \mathrm{SD}$ of three independent experiments. *Student's $t$-test.

subpopulation of laryngeal cancer TU212 and TU686 cells, and $\alpha 2 \delta 1^{+}$LSCC cells possessed stem cell-like properties.

Immunofluorescence staining of 16 LSCC specimens showed that $\alpha 2 \delta 1^{+}$was significantly more frequently expressed in laryngeal cancer tissue than that in paracancerous tissues and normal tissues. This result is consistent with previous findings in other malig- nancies $[10,13]$. Next, we used qPCR to detect CSCassociated genes and drug resistance genes. The results showed high level of expression of stem cell-associated genes, including BMI-1 [4], SOX2 [27], ЕpCAM [28], OCT4 [29], CTNNB1 [30], and ABCG2 [31-33] in $\alpha 2 \delta 1^{+}$TU686 cells. These data together showed that a CSC subpopulation in laryngeal cancer is marked by a surface phenotype $\alpha 2 \delta 1$. Only $\alpha 2 \delta 1^{+}$cells for this 


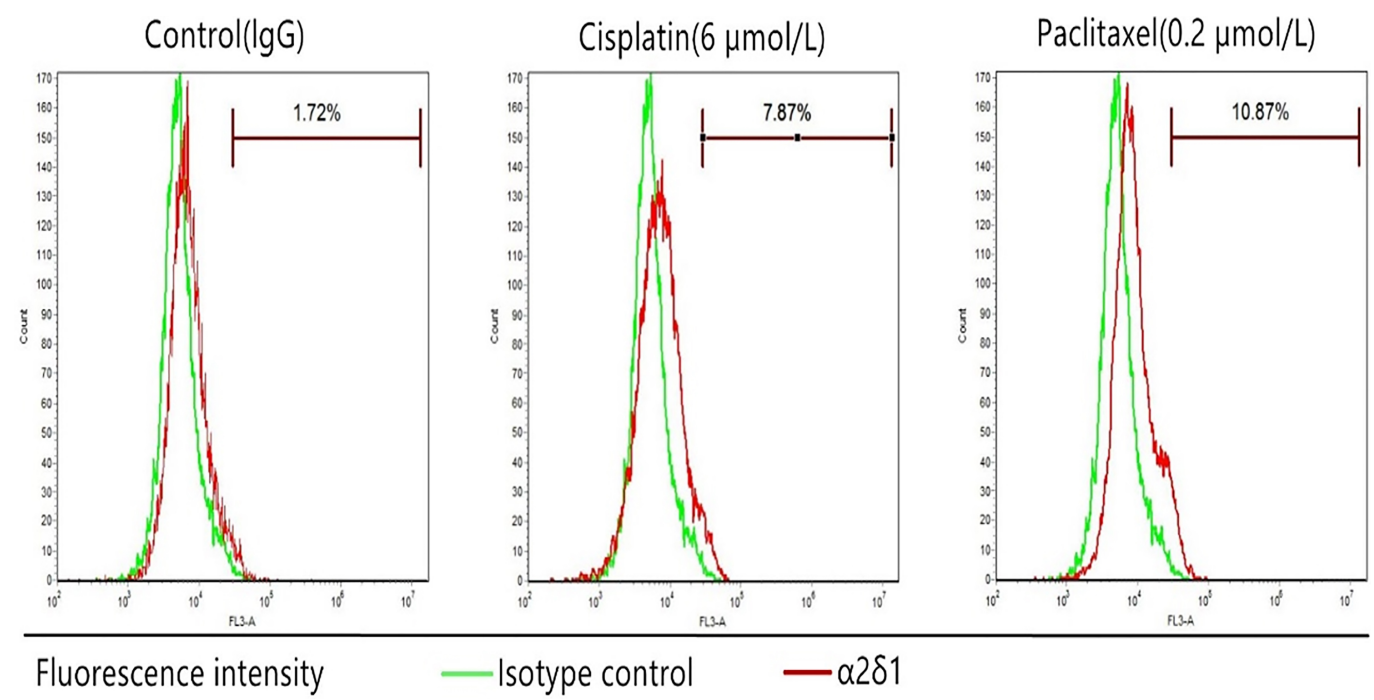

Fig. 6. Chemoresistance ofTU686 cells. The percentage of sorted $\alpha 2 \delta 1^{+}$TU686 cells after exposure to cisplatin $(6 \mu$ mol/L) or paclitaxel $(0.2 \mu \mathrm{mol} / \mathrm{L})$ for 72 hours.

marker showed stem-cell like characteristics, including the ability to differentiate and for self-renewal, and the capability of resisting standard chemotherapy.

Next, we showed that TU212 and TU686 cells also contain a $\alpha 2 \delta 1^{+}$subpopulation. Purified $\alpha 2 \delta 1^{+}$cells had the capability to multiply as spheroids in growth factor-supplemented, serum-free medium under nonadherent conditions. After culturing for 4 weeks, cancer spheres of undifferentiated $\alpha 2 \delta 1^{+}$cells were obtained. In contrast, the sphere-forming ability of $\alpha 2 \delta 1^{-}$cells were much weaker than $\alpha 2 \delta 1^{+}$cells under the same conditions. Cell spheres deriving from single cells that were acquired from dissociated $\alpha 2 \delta 1^{+}$cells showed nearly limitless growth capability and could be expanded in subsequent serial propagations. Therefore, we speculate that $\alpha 2 \delta 1$ may play a crucial role in the sphere formation ability of TU212 and TU686 cells. This is consistent with a previous study [10], which found that $\alpha 2 \delta 1 \mathrm{Cav}$ channel overexpression resulted in increased sphere formation in HCC Hep-11 and Hep-12 cells. Self-renewal ability is one of the characteristics of CSC; these results indicate that $\alpha 2 \delta 1^{+}$cells in TU212 and TU686 cells may represent CSCs.

We next evaluated the differentiation and migration/invasion potential of the $\alpha 2 \delta 1^{+}$subpopulation in TU212 and TU686 cells. The results showed that $\alpha 2 \delta 1^{+}$cells could differentiate into $\alpha 2 \delta 1^{-}$cells and $\alpha 2 \delta 1^{+}$cells while $\alpha 2 \delta 1^{-}$cells did not have this property. $\alpha 2 \delta 1^{+}$cells also had greater differentiation and migration/invasion potential than $\alpha 2 \delta 1^{-}$cells. The ability to differentiate and migrate/invade is also a feature of CSCs.
Consistent with a previous study on $\alpha 2 \delta 1^{+}$cells in lung cancer [13], treatment with cisplatin and paclitaxel increased the percentage of $\alpha 2 \delta 1^{+}$cells in TU686 cells in our experiments, suggesting that laryngeal cancer cells expressing $\alpha 2 \delta 1$ are resistant to chemotherapeutic agents. According to previous report [13], we believe that cisplatin/paclitaxel may cause $\alpha 2 \delta 1^{-}$cells to undergo apoptosis or necrosis, while $\alpha 2 \delta 1^{+}$cells are insensitive to chemotherapeutic drugs and can survive the tumoricidal effect of cisplatin/paclitaxel. Therefore, the ratio of $\alpha 2 \delta 1^{+}$cells to all viable cells increases, and the effect of enriching CSC is achieved. In future studies, we will verify the above speculation and grading the concentration of chemotherapeutic drugs to see if the change in the ratio of $\alpha 2 \delta 1^{+}$cells is timeand dose-dependent in a range of drug concentrations. Available evidence indicates that $\alpha 2 \delta 1^{+}$cells are resistant to cisplatin/paclitaxel. Resistance to radiotherapy and chemotherapy is one of the important biological characteristics of CSCs.

Previous studies have confirmed that $\alpha 2 \delta 1$ plays a crucial role in regulating the amplitude of CSC calcium oscillations [10]. Usually, spontaneous calcium oscillations in cells are caused by excitable tissues such as muscles, nerve tissues, embryonic stem cells, and immature dendritic cells. In most cases, their regulatory mechanisms and biological functions have not been elucidated. Calcium oscillations, a major form of calcium signaling, can promote the expression of specific genes, which is related to the magnitude and duration of calcium transients. It is possible to bind to the en- 


\begin{tabular}{|c|c|c|}
\hline Group & TU212 $\alpha 2 \delta 1^{+}$ & TU212 $\alpha 2 \delta 1^{-}$ \\
\hline $10^{3}$ & $5 / 5$ & $3 / 5$ \\
\hline $10^{2}$ & $5 / 5$ & $2 / 5$ \\
\hline CSC frequency & $1 / 1$ & $1 / 711$ \\
\hline $95 \% \mathrm{CI}$ & $1 / 125-1 / 1$ & $1 / 1938-1 / 362$ \\
\hline P value & \multicolumn{2}{|c|}{$3.32 \mathrm{E}-5$} \\
\hline
\end{tabular}

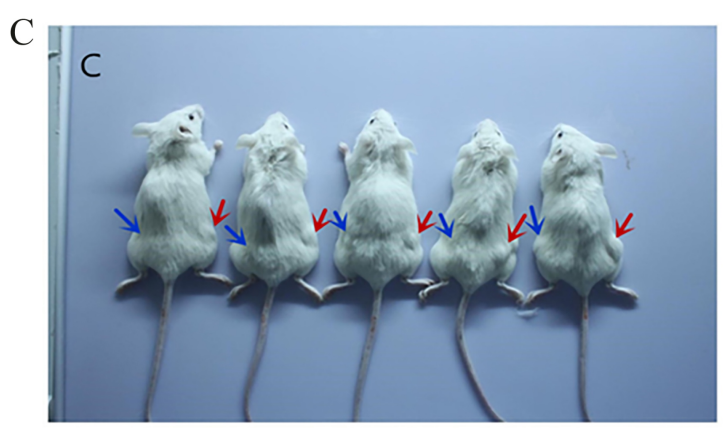

E
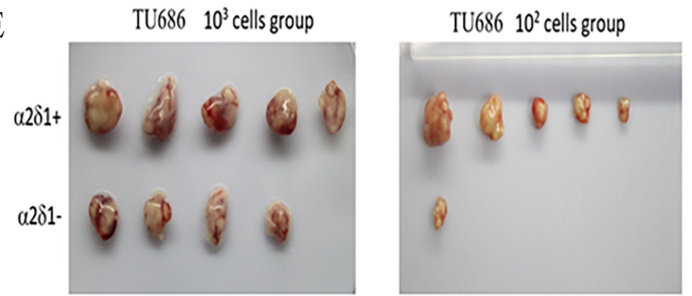

G
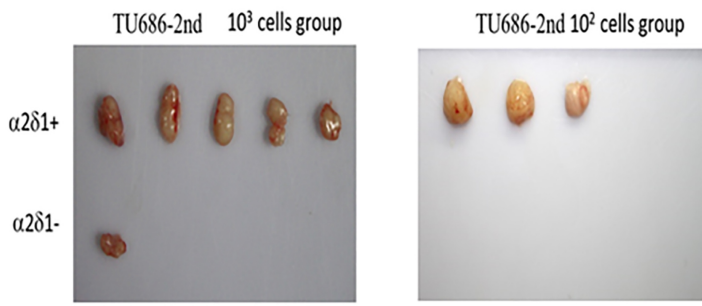

I

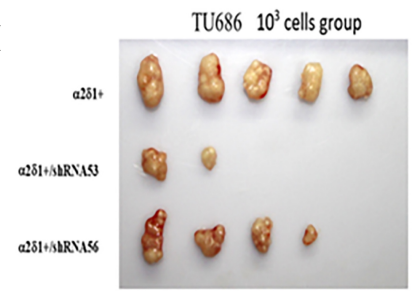

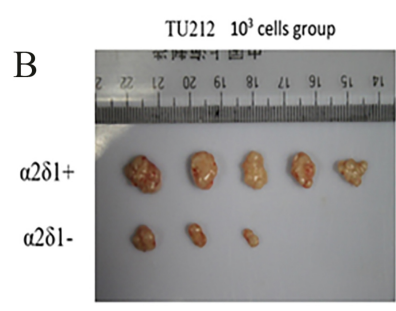

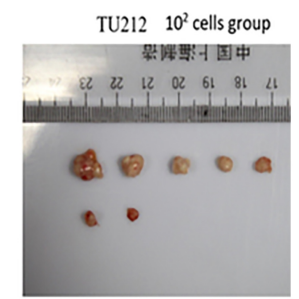

\begin{tabular}{ccc}
\hline D roup & TU686 $0.281^{+}$ & TU686 $\alpha 281^{\circ}$ \\
\hline $10^{3}$ & $5 / 5$ & $4 / 5$ \\
$10^{2}$ & $5 / 5$ & $1 / 5$ \\
CSC frequency & $1 / 1$ & $1 / 580$ \\
$95 \%$ CI & $1 / 125-1 / 1$ & $1 / 1543-1 / 218$ \\
P value & \multicolumn{2}{c}{$1.22 \mathrm{E}-4$} \\
\hline
\end{tabular}

F

\begin{tabular}{ccc}
\hline Group & TU686-2nd $\alpha 281^{+}$ & TU686-2nd $0281^{-}$ \\
\hline $10^{3}$ & $5 / 5$ & $1 / 5$ \\
$10^{2}$ & $3 / 5$ & $0 / 5$ \\
CSC frequency & $1 / 109$ & $1 / 4983$ \\
$95 \%$ CI & $1 / 348-1 / 34$ & $1 / 35191-/ 705$ \\
P value & \multicolumn{2}{c}{$4.37 \mathrm{E}-5$} \\
\hline
\end{tabular}

\begin{tabular}{|c|c|c|c|}
\hline Group & $\omega_{2}^{2} \delta 1^{+} /$Control & $\omega 281^{+} / \mathrm{shRNA53}$ & $\omega 281^{+} / \operatorname{shRNA56}$ \\
\hline $10^{3} \mathrm{TU} 686$ cells & $5 / 5$ & $2 / 5$ & $4 / 5$ \\
\hline $10^{2} \mathrm{TU} 686$ cells & $5 / 5$ & $2 / 5$ & $3 / 5$ \\
\hline CSC Frequency & $1 / 1$ & 1/1061 & $1 / 360$ \\
\hline $95 \% \mathrm{CI}$ & $1 / 125-1$ & $1 / 31281-1 / 360$ & 1/988-131 \\
\hline Pvalue & & $4.67 \mathrm{E}-6$ & 0.000758 \\
\hline
\end{tabular}

$\mathrm{J}$

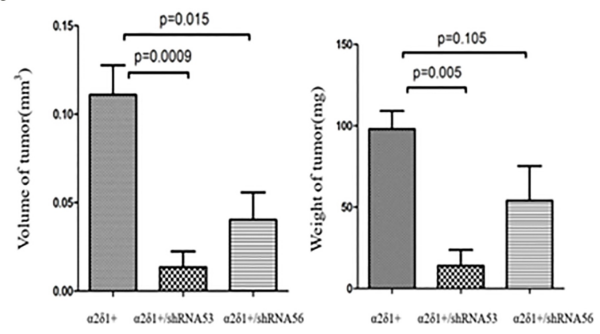

Fig. 7. Tumorigenesis of $\alpha 2 \delta 1^{+}$vs. $\alpha 2 \delta 1^{-}$subpopulation. (A) TU212 cells were transplanted subcutaneously in NOD/SCID mice as indicated. (B, C) Representative photographs showing dissected tumors by FACS-purified $\alpha 2 \delta 1^{+}$(red arrows) and $\alpha 2 \delta 1^{-}$(blue arrows) TU212 cells. (D) TU686 cells were inoculated subcutaneously in NOD/SCID mice as indicated. (E) Photographs showing dissected tumors formed by FACS-purified $\alpha 2 \delta 1^{+}$and $\alpha 2 \delta 1^{-}$TU686 cells. (F, G) Serial inoculation of $10^{2}$ and $10^{3}$ TU686 cells into NOD/SCID mice from tumors formed by $\alpha 2 \delta 1^{+}$ TU686 cells. (H, I) Tumorigenicity of TU686 $\alpha 2 \delta 1^{+}$cells FACS-purified from indicated sources after $\alpha 2 \delta 1$ shRNA53/shRNA56 knockdown. TU686 $\alpha 2 \delta 1^{+}$cells were incubated with indicated lentiviruses for 4 hours, and $10^{2} / 10^{3}$ cells per mouse were injected. The statistical results of tumorigenic formation in 5 mice were calculated. (J) The volume and weight of tumors by $\alpha 2 \delta 1^{+}$cells, and $\alpha 2 \delta 1^{+}$cells knockdown with shRNA53and shRNA56 were detected. 
hancer site and initiate transcription by keeping the transcription factor in the nucleus at a sufficiently high level. We hypothesize that $\alpha 2 \delta 1$ may be involved in the "amplitude-encoding" signal in LSCC, thereby maintaining the stem cell characteristics of LSCC.

Upon in vivo tumor xenograft assays, 100 purified $\alpha 2 \delta 1^{+}$cells were sufficient for tumor formation. In contrast, $\alpha 2 \delta 1^{-}$cells either were completely nontumorigenic or formed tiny nodules only occasionally. In vivo serial inoculation assays with re-sorted cells from tumors formed by implanted $\alpha 2 \delta 1^{+}$cells confirmed the tumorigenesis potential of $\alpha 2 \delta 1^{+}$but not $\alpha 2 \delta 1^{-}$cells. Knocking down $\alpha 2 \delta 1$ in purified $\alpha 2 \delta 1^{+}$ cells using shRNA reduced sphere formation and decreased tumorigenic cells in TU686 cells. These data indicate that $\alpha 2 \delta 1$ has an important influence on sphere formation or tumorigenicity of LSCC both in vivo and in vitro, and has the potential to become a LSCC CSC marker. Nevertheless, the role of $\alpha 2 \delta 1$ in the onset, progression and metastasis of LSCC remains to be further studied.

\section{Conclusion}

In summary, the results from the current study indicated the presence of a $\alpha 2 \delta 1^{+}$subpopulation with CSC-like property in laryngeal cancer. The specific mechanism of CSC-like effects exerted by $\alpha 2 \delta 1$ in laryngeal cancer requires further study.

\section{Acknowledgments}

This research was supported by the Scientific Research Common Program of Beijing Municipal Commission of Education (\#KM201510025028) and Research Foundation of Beijing Friendship Hospital, Capital Medical University (\#yydszx2015-02, \#yyqdkt 2014-23).

\section{Conflict of interest}

The authors declare that they do not have any potential conflict of interests in relation to the contents of this manuscript.

\section{References}

[1] R. Siegel, E. Ward, O. Brawley and A. Jemal, Cancer statistics, 2011: The impact of eliminating socioeconomic and racial disparities on premature cancer deaths, CA: A Cancer Journal for Clinicians 61 (2011), 212-236.
[2] Y. Liu, Q. Zhao, G. Ding, Y. Zhu, W. Li, W. Chen and J. Chin, Incidence and mortality of laryngeal cancer in China, 20082012, Cancer Res 30 (2018), 299-306.

[3] L. Du, H. Li, C. Zhu, R. Zheng, S. Zhang and W. Chen, Incidence and mortality of laryngeal cancer in China, 2011, Chinese J Cancer Res 27 (2015), 52-58.

[4] D. Yu, Y. Liu, J. Yang, C. Jin, X. Zhao, J. Cheng, X. Liu and X. Qi, Clinical implications of BMI-1 in cancer stem cells of laryngeal carcinoma, Cell Biochem Biophys 71 (2015), 262269.

[5] M. Martín, A. Hinojar, L. Cerezo, J. García, M. Lopez, J. Prada, A. Marín and C. Gamallo, Aldehyde dehydrogenase isoform 1 (ALDH1) expression as a predictor of radiosensitivity in laryngeal cancer, Clinical and Translational Oncology 18 (2016), 825-830.

[6] J. Wang, Y. Wu, W. Gao, F. Li, Y. Bo, M. Zhu, R. Fu, Q. Liu, S. Wen and B. Wang, Identification and characterization of $\mathrm{CD} 133^{+} \mathrm{CD} 44^{+}$cancer stem cells from human laryngeal squamous cell carcinoma cell lines, J Cancer 8 (2017), 497506.

[7] M.C. de Jong, J. Pramana, J.E. van der Wal, M. Lacko, C.J. Peutz-Kootstra, J.M. de Jong, R.P. Takes, J.H. Kaanders, B.F. van der Laan, J. Wachters, J.C. Jansen, C.R. Rasch, M.L. van Velthuysen, R. Grénman, F.J. Hoebers, E. Schuuring, M.W. van den Brekel and A.C. Begg, CD44 expression predicts local recurrence after radiotherapy in larynx cancer, Clin Cancer Res 16 (2010), 5329-5338.

[8] M. Baumann and M. Krause, CD44: A cancer stem cellrelated biomarker with predictive potential for radiotherapy, Clin Cancer Res 16 (2010), 5091-5093.

[9] I. Suer, O.F. Karatas, B. Yuceturk, M. Yilmaz, G. Guven, O. Buge, H. Cansiz and M. Ozen, Characterization of stem-like cells directly isolated from freshly resected laryngeal squamous cell carcinoma specimens, Curr Stem Cell Res Ther 9 (2014), 347-353.

[10] W. Zhao, L. Wang, H. Han, K. Jin, N. Lin, T. Guo, Y. Chen, H. Cheng, F. Lu, W. Fang, Y. Wang, B. Xing and Z. Zhang, 1B50-1, a mAb raised against recurrent tumor cells, targets liver tumor-initiating cells by binding to the calcium channel $\alpha 2 \delta 1$ subunit, Cancer Cell 23 (2013), 541-556.

[11] B. Sainz and C. Heeschen, Standing out from the crowd: Cancer stem cells in hepatocellular carcinoma, Cancer Cell $\mathbf{2 3}$ (2013), 431-433.

[12] Y. Zhang, W. Zhao, H. Han, S. Li, D. Chen and Z. Zhang, MicroRNA-31 suppresses the self-renewal capability of $\alpha 2 \delta 1^{+}$liver tumor-initiating cells by targeting ISL1, Oncotarget 8 (2017), 87647-87657.

[13] J. Yu, S. Wang, W. Zhao, J. Duan, Z. Wang, H. Chen, Y. Tian, D. Wang, J. Zhao, T. An, H. Bai, M. Wu and J. Wang, Mechanistic exploration of cancer stem cell marker voltage-dependent calcium channel $\alpha 2 \delta 1$ subunit-mediated chemotherapy resistance in small-cell lung cancer, Clin Cancer Res 24 (2018), 2148-2158.

[14] S. Zarei, A.A. Bayat, R. Hadavi, A.R. Mahmoudi, B. Tavangar, Y. Vojgani, M. Jeddi-Tehrani and Z. Amirghofran, Production and characterization of a peptide-based monoclonal antibody against CD44 variant 6, Monoclonal Antibodies in Immunodiagnosis and Immunotherapy 34 (2015), 36-43.

[15] C. Zhao, Z.G. Ma, S.L. Mou, Y.X. Yang, Y.H. Zhang and W.C. Yao, Targeting effect of microRNA on CD133 and its impact analysis on proliferation and invasion of glioma cells, Genetics and Molecular Research 16 (2017), 1-9.

[16] S.K.M. Li, D.K. Smith, W.Y. Leung, A.M.S. Cheung, E.W.F. Lam, G.P. Dimri and K.M. Yao, FoxM1c counteracts oxida- 
tive stress-induced senescence and stimulates bmi-1 expression, J Biol Chem 283 (2008), 16545-16553.

[17] S. Klein, J.L. Dvornik, A.R. Yarrabothula and C. Schaniel, A Marfan syndrome human induced pluripotent stem cell line with a heterozygous FBN1 c.4082G > A mutation, ISMMSi002-B, for disease modeling, Stem Cell Res $\mathbf{2 3}$ (2017), 73-76.

[18] D.J. Eastburn, A. Sciambi and A.R. Abate, Picoinjection enables digital detection of RNA with droplet rt-PCR, Plos One 8 (2013), e62961.

[19] R. Kim and J. Nam, OCT4 expression enhances features of cancer stem cells in a mouse model of breast cancer, Lab Anim Res 27 (2011), 147.

[20] J. Durand, A. Lampron, T.L. Mazzuco, A. Chapman and I. Bourdeau, Characterization of differential gene expression in adrenocortical tumors harboring $\beta$-catenin (CTNNB1) mutations, J Clin Endocrinol Metab 96 (2011), E1206-E1211.

[21] D.A. Evseenko, J.W. Paxton and J.A. Keelan, The xenobiotic transporter ABCG2 plays a novel role in differentiation of trophoblast-like BeWo cells, Placenta 28 (2007), S116-S120.

[22] F. Marchesi, P. Monti, B.E. Leone, A. Zerbi, A. Vecchi, L. Piemonti, A. Mantovani and P. Allavena, Increased survival, proliferation, and migration in metastatic human pancreatic tumor cells expressing functional CXCR4, Cancer Res 64 (2004), 8420-8427.

[23] M.W. Pfaffl, A new mathematical model for relative quantification in real-time RT-PCR, Nucleic Acids Res 29 (2001), e45

[24] L. Lan, H. Han, H. Zuo, Z. Chen, Y. Du, W. Zhao, J. Gu and Z. Zhang, Upregulation of myosin Va by Snail is involved in cancer cell migration and metastasis, Int J Cancer 126 (2010), 53-64.

[25] J. Wu, Z. Yan, Z. Li, X. Qian, S. Lu, M. Dong, Q. Zhou and N. Yan, Structure of the voltage-gated calcium channel Cav1.1 at $3.6 \AA$ resolution, Nature 537 (2016), 191-196.
[26] F. Van Petegem, K.A. Clark, F.C. Chatelain and D.L. Minor, Structure of a complex between a voltage-gated calcium channel $\beta$-subunit and an $\alpha$-subunit domain, Nature 429 (2004), 671-675.

[27] L. Hui, J. Zhang, X. Ding, X. Guo and X. Jiang, Matrix stiffness regulates the proliferation, stemness and chemoresistance of laryngeal squamous cancer cells, Int J Oncol $\mathbf{5 0}$ (2017), 1439-1447.

[28] N. Murakami, T. Mori, S. Yoshimoto, Y. Ito, K. Kobayashi, H. Ken, M. Kitaguchi, S. Sekii, K. Takahashi, K. Yoshio, K. Inaba, M. Morota, M. Sumi and J. Itami, Expression of EpCAM and prognosis in early-stage glottic cancer treated by radiotherapy, Laryngoscope 124 (2014), e431-436.

[29] N.M.F. El Deeb and E. Abdelzaher, Stem cell markers OCT4 and nestin in laryngeal squamous cell carcinoma and their relation to survivin expression, Pathol Res Pract 210 (2014), $751-758$.

[30] P. Machin, L. Catasus, C. Pons, J. Muñoz, X. Matias-Guiu and J. Prat, CTNNB1 mutations and $\beta$-catenin expression in endometrial carcinomas, Hum Pathol 33 (2002), 206-212.

[31] B. Shen, D. Li, P. Dong and S. Gao, Expression of ABC transporters is an unfavorable prognostic factor in laryngeal squamous cell carcinoma, Ann Otol Rhinol Laryngol 120 (2011), $820-827$.

[32] X. Qi, D. Yu, B. Jia, C. Jin, X. Liu, X. Zhao and G. Zhang, Targeting $\mathrm{CD}_{133}{ }^{+}$laryngeal carcinoma cells with chemotherapeutic drugs and siRNA against ABCG2 mediated by thermo/pH-sensitive mesoporous silica nanoparticles, $\mathrm{Tu}$ mor Biol 37 (2016), 2209-2217.

[33] Y.J. Pu, L. Yan, Z. Wei, Y. Dan, W. Lian-Ji, J.C. Shun, Chemoresistance of $\mathrm{CD} 133^{+}$cancer stem cells in laryngeal caronoma, Chin Med J (Engl) 124 (2011), 1055-1060. 